\title{
Pabellón de reposo, de Camilo José Cela, a la luz de las reflexiones sobre el papel de las narrativas patográficas en la curación del enfermo de tuberculosis
}

\author{
Camilo José Cela's Pabellón de reposo \\ in the Light of the Reflections on the Role \\ of Pathographic Narratives in Healing \\ the Patient with Tuberculosis
}

RaQuel VELÁZQUEZ VELÁZQUEZ

RECIBIDO: 12 DE JUNIO DE 2016 ACEPTADO: 23 DE AGOSTO DE 2016

Departamento de Filología Hispánica

Universidad de Barcelona

Avda. Gran Via de les Corts Catalanes, 585. Barcelona, 08007

raquel_velazquez@ub.edu

Orcid ID: 0000-0002-9507-0065

Resumen: Si bien contamos con análisis detenidos y pormenorizados de la novela Pabellón de reposo, que atienden a la estructura, la temporalidad, o la configuración de los personajes, la crítica ha soslayado el valor de la novela de Camilo José Cela en tanto que narrativa patográfica. Este trabajo se ocupa de Pabellón de reposo en su calidad de representación de la enfermedad de la tuberculosis, la vida de los enfermos y el papel que cumplen sus discursos patográficos. En primer lugar, se estudia cómo, a pesar de que en la primera mitad del siglo xx ya ha desaparecido prácticamente la imagen glamurosa de la tuberculosis, Cela opta por una representación tamizada de la enfermedad, donde la herencia romántica cala en sus personajes enfer- mos, y la novela se tiñe de un romanticismo que la literatura sanatorial había ya dejado atrás. En segundo lugar, se ponen en relación algunas de las opiniones médicas de la época con las reflexiones que el autor incluye en Pabellón de reposo acerca del papel que puede cumplir la escritura o lectura de narrativas patográficas en la mejoría o empeoramiento del enfermo. Se examina así la existencia en la primera mitad del siglo xx de esta conexión entre Medicina y Humanidades que constituye hoy el centro de interés de la disciplina de las Medical Humanities.

Palabras clave: Pabellón de reposo. Camilo José Cela. Narrativa patográfica. Tuberculosis. Sanatorio. 
Abstract: Although we have detailed and in-depth analysis about the novel Pabellón de reposo, dealing with structure, temporality, or configuration of the characters, academics have sidestepped the value of Camilo José Cela's novel as pathographic narrative. This paper attends to Pabellón de reposo as a representation of ТВ disease, the life of patients, and the role played by their discourses on their illness. First, we argue how, even though the glamorous image of tuberculosis was almost gone in the first half of the twentieth century, Cela opts for a sifted representation of the disease, where the romantic heritage has a deep impact on his ill characters, and the novel is tinged with the romanticism that litera- ture of sanatoria had already left behind. Second, we establish a connection between some of the medical opinions of the time and the reflections that the author includes in Pabellón de reposo about whether the writing or reading of pathographic narratives can help to improve or worsen the health of the patient. In this way, we review the existence in the first half of the twentieth century of this connection between Medicine and Humanities, which is nowadays the focus of the discipline of Medical Humanities.

Keywords: Pabellón de reposo. Camilo José Cela. Pathographic Narrative. Tuberculosis. Sanatorium.

A finales del siglo XIX, pero sobre todo en la primera mitad del siglo XX asoman con profusión una serie de relatos que conforman lo que podría denominarse la literatura sanatorial, y que giran en torno a las narrativas patográficas que se producen en el seno de ese microcosmos. Si bien la literatura había reflejado ya los efectos devastadores que la "peste blanca" estaba causando en Europa, lo había hecho mostrando una gran distancia entre la representación literaria y la realidad terrorífica que biológicamente vivía el enfermo. Algunos médicos habían alzado ya sus voces en el siglo XVIII acerca del flaco favor que hacía la idealización y estilización de la enfermedad de la consunción, tan lejos de la buena y dulce muerte que pintaban los poetas; ${ }^{2} \mathrm{y}$, sin embargo, no es hasta finales del siglo XIX, coincidiendo con el descubrimiento en 1882 del bacilo causante de la tuberculosis (y, por tanto, de su carácter de infección bacteriana), cuando la tisis pulmonar empieza a desprenderse de su glamur y comienzan las reacciones hacia la representación romántica de la enfermedad (que aun así dejará algunos rescoldos incluso en la narrativa ficcional del propio Cela). En España, uno de los testimonios que se rebelará contra esta imagen idílica de los tuberculosos será Rosalía de Castro, quien en una carta de diciembre de 1861 dirigida a Manuel Murguía, exponía:

1. En 1861 Oliver Wendell Holmes (352) se refiere a la enfermedad como "the white plague of the north". Ver Dubos/Dubos; Madkour (20-21).

2. Para un estudio de las relaciones entre consunción y literatura (principalmente en lengua inglesa), y la representación de la enfermedad de la tuberculosis hasta el siglo XIX, ver la completa e imprescindible obra de Clark Lawlor. 
Si en realidad llegase a ponerme tísica, lo único que querría es acabar pronto, porque moriría medio desesperada al verme envuelta en gargajos, y cuanto más durase el negocio, peor. ¿Quién demonio habrá hecho de la tisis una enfermedad poética? (s.p.)

En 1894 el narrador de "El dúo de la tos", relato de Leopoldo Alas, ofrecería una nueva muestra de esta situación revertida al apuntar: "Ya había pasado el romanticismo que había tenido alguna consideración con los tísicos" (571).

Era comprensible esta actitud de protesta teniendo en cuenta la magnitud social, nada alentadora, que estaba cobrando la enfermedad en toda Europa, lo cual obligó a muchos países, en lo que era casi una campaña internacional, a crear instituciones de lucha antituberculosa a nivel estatal, ligadas a tareas de concienciación, y especialmente a la construcción de sanatorios y dispensarios (Bryder 15-16). En España, donde en el paso de los años treinta a los cuarenta, la tisis mataba cada año a más de 30000 enfermos, ${ }^{3}$ la tuberculosis tiene una presencia continua en la sociedad. El Patronato Nacional Antituberculoso (PNA), fundado en diciembre de 1936 por el bando sublevado, pero herencia de proyectos anteriores, ${ }^{4}$ convoca concursos de arquitectura para la construcción de los nuevos sanatorios que intentarán proporcionar al menos una cama por defunción, y que se publicitarán más tarde recurriendo a la promesa del reposo y otras terapias necesarias para la curación. Por su parte, la prensa (basta echar una hojeada al Blanco y negro de las décadas treinta y cuarenta) se hace eco de las conferencias y talleres impartidos sobre nuevos tratamientos, y abundan en sus páginas tanto los reportajes a médicos españoles y extranjeros que disertan sobre el diagnóstico y las posibles curas, como

3. Ver Navarro García 223. Un estudio encabezado por Abilio Burgos de Pablo (160-61) a principios de los ochenta da una media de 25000 defunciones en la década de los cuarenta, con datos tomados de la Delegación Provincial del Instituto Nacional de Estadística. El lector interesado encontrará en los volúmenes de Pieltáin Álvarez-Arenas y Ruiloba Quecedo un extenso análisis de la situación de la tuberculosis en España en la primera mitad del siglo XX, en estrecha relación con la arquitectura sanatorial.

4. El rey Alfonso XIII creó junto a su esposa Victoria Eugenia el Real Patronato Central de Dispensarios e Instituciones Antituberculosas en 1907 por orden del Ministro de Gobernación, Juan de la Cierva $(A B C 18$ diciembre 1907, 2). Se inició como fundación privada, y el Estado participaba como un donante más (ver Pieltáin Álvarez-Arenas y Ruiloba Quecedo). El primer gobierno republicano en 1931 decide estatalizar la lucha contra la tuberculosis, encomendando la organización antituberculosa a la Dirección General de Sanidad, previa disolución del Real Patronato creado por Primo de Rivera en 1924. Se dota a la campaña de un presupuesto para iniciar la construcción de dispensarios y sanatorios, y se empieza a desarrollar la idea de la implantación de un seguro general de enfermedad, frustrado a causa del levantamiento de 1936 (Molero Mesa 1994, 201-03). 
los anuncios que ofrecen remedios milagrosos (el Jarabe de Hipofosfitos Salud, las pastillas Aspaime) que igual curan la anemia que la tuberculosis.

En este contexto, los escritores dan un nuevo giro a la literatura patográfica que parte de la experiencia más o menos directa con la tuberculosis. En general, estas narrativas (y entiendo narrativa en el sentido más amplio del término, como historia que el paciente se cuenta a sí mismo o a los demás; es decir, como la transformación en discurso que el yo tuberculoso realiza de su enfermedad) se basan en un contacto previo del escritor con la enfermedad, ya sea como pariente, amigo o testigo, en el caso de Thomas Mann (quien visita a su mujer en el sanatorio suizo de Davos); como médico, en el caso de Valdés Lambea (director del sanatorio de Hoyos durante la estancia de Cela); o, naturalmente, como enfermo, dando lugar a autopatografías o autoficciones más o menos veladas de uno o más "yoes", como en el caso de Gesualdo Bufalino, de Camilo José Cela, o de Blai Bonet.

De cualquier modo, todas ellas son reflejo de una realidad incuestionable, y en ese sentido, independientemente del poso autobiográfico que puedan contener, deben concebirse como representaciones. Por ello, mi intención en este trabajo es ocuparme de Pabellón de reposo precisamente en su calidad de representación, que aun escrita en el siglo XX surge teñida de la imagen romántica de la enfermedad. La concepción de la novela de Cela como representación patográfica nos lleva a centrarnos asimismo en las reflexiones del autor -traspasadas a la novela- acerca del papel que la escritura o la lectura de narrativas de su enfermedad puede cumplir en la mejoría o empeoramiento del enfermo, discusión que en la segunda mitad del siglo Xx promovió la creación, especialmente en Estados Unidos y el Reino Unido (Whitehead 108-09), de una disciplina autónoma como las Medical Humanities.

Para un análisis detenido y pormenorizado de la novela de Cela contamos con los clásicos estudios, aún vigentes, de Paul Ilie, David W. Foster, Robert Kirsner, José María Castellet, Zamora Vicente, D. W. McPheeters, John W. Kronik o Eugenio García de Nora, entre otros, que nos ofrecen análisis exhaustivos centrados en la estructura y la temporalidad de la novela, siempre dentro de los límites de Pabellón de reposo. A ellos remito al lector. Al entender la obra de Cela del 43 fundamentalmente como representación de la enfermedad de la tuberculosis, este trabajo intenta salirse de esos límites para detenerse en la potencial influencia de las narrativas tuberculosas en la construcción de la mitología cultural de la enfermedad, tanto en el seno de la obra, como en la sociedad española de los años cuarenta. 
Si bien cabe mencionar la originalidad de la perspectiva biopolítica con la que en 2015 Óscar Pérez propone leer Pabellón de reposo, resulta cuestionable, a la luz de los datos, la relación demasiado directa que el crítico establece entre el tratamiento de la enfermedad de la tuberculosis y los discursos biopolíticos franquistas (Pérez 232). Aunque ciertamente la dictadura de Franco inicia toda una serie de medidas de control sobre la enfermedad (publicación de la Ley de Bases de Sanidad Nacional en 1944, instauración del PNA), se trata de un control estatal que -como hemos aducido ya- no es exclusivo del gobierno franquista ni se circunscribe únicamente a España. El argumento principal con el que Pérez apoya su aproximación biopolítica a la novela de Cela se basa en el proceso de deshumanización del enfermo que -a su juicio- establece el gobierno franquista al centrar sus discursos en torno a las camas necesarias, las camas existentes o las camas por construir, en lugar de hablar de enfermos, pacientes y víctimas mortales (233). En opinión de Pérez, Pabellón de reposo se hace eco de este "acto de ejercicio de poder" con el que se "arrebata la identidad de los enfermos" (235) cuando Cela nombra con un número a los pacientes de su sanatorio. No obstante, esta conexión que -de acuerdo con Pérez- "es quizá el elemento que permite realizar esta aproximación [biopolítica a la novela] con mayor claridad" (237), aun siendo oportuna, es extrapolable a otros países y a otras narrativas sanatoriales que constituyen representaciones de la enfermedad, algo que revela rápidamente un acercamiento comparatista. Por un lado, los discursos y las estadísticas sobre el número de camas necesarias son compartidos en toda Europa, en una tendencia a centrarse en la solución más que en el problema, y surgen con anterioridad al periodo franquista (ver, por ejemplo, la sesión del 27 de marzo de 1925 de los Anales de la Real Academia de Medicina y Cirugía de Barcelona). Por otro lado, como avanzábamos, la propensión a referirse a los enfermos sanatoriales por el número de habitación afecta no solo a Pabellón de reposo, sino a otras narrativas sanatoriales no contextualizadas en España o ajenas al ejercicio de poder franquista, como puede ser el relato de Roberto Arlt "Ester Primavera" (1928), ambientado en Buenos Aires; o el de Clarín, "El dúo de la tos", publicado en 1894. En su artículo, Pérez defiende asimismo que con el franquismo se estableció, "desde la primera mitad de la década de los cuarenta" (233), una conexión entre tuberculosis y sexualidad que estigmatizaba moralmente al enfermo. Sin embargo, de nuevo, esa relación -existente, en efecto, en algunos círculos médicos- no es exclusiva del franquismo ni de la España de posguerra, sino que 
responde a un intento generalizado de afrontar el desconocimiento sobre las vías de infección de la tuberculosis, que llevaban a relacionarla con las enfermedades de transmisión sexual. El doctor especialista Cortejoso dedicaba, por ejemplo, en 1939 un capítulo de su obra La enfermera en la lucha antituberculosa al vínculo entre tuberculosis y prostitución, atribuyendo a la vida disipada (enfermedades venéreas, alcoholismo, tabaquismo) una fuente directa de contagio. Estas correlaciones a las que se ha referido detenidamente en varios de sus trabajos Molero Mesa (1989; 1994, no contemplados por Pérez) se remontan a la segunda mitad del siglo XIX, cuando ya entonces se incluían, en congresos españoles de tuberculosis, el onanismo y la homosexualidad como causas responsables de la tisis en la juventud (Molero Mesa 1989, 221). Pérez soslaya, además, los estudios sociológicos, con Goffman a la cabeza, sobre la estigmatización del enfermo o sobre los centros psiquiátricos y sanatoriales que explican en gran medida el funcionamiento de estos lugares heterotópicos, según terminología de Foucault, más allá del peso del contexto político.

El presente trabajo se aleja de esa posible vertiente biopolítica de la narrativa sanatorial celiana suscitada por Pérez para ocuparse, por el contrario, de los términos en que se produce la representación de la enfermedad que constituye Pabellón de reposo y, en general, cualquier narrativa sanatorial.

La idea de representación subyace y se apoya en la decisión por parte de Cela de construir una novela en la que -para subrayar la atmósfera de atemporalidad, de no tiempo- se ha evitado toda "referencia geográfica, onomástica o temporal que permitiese su localización en una época o lugar determinados (salvo, quizá, la relativa, y siempre muy aproximada, situación en el calendario que pudiera averiguarse por las terapéuticas empleadas con mis marionetas)" (Cela 1958, 11). Este anonimato y la ausencia de detalles que identifiquen la realidad intratextual con la extratextual permiten que el denominado por Cela "mi experimento pacífico" (Cela 1958, 11) tienda a la generalización, buscando desenmascarar "la circunstancia del tiempo que la constriñe y del espacio que la atenaza" (Cela 1965, 9), según nos avisa Cela en la nota a la primera edición (fechada en diciembre de 1943). De este modo, se produce la sensación de que, desenmarcada de tiempo y espacio concretos, estamos ante la representación de cualquier tísico en cualquier pabellón.

Resulta obvio que no debe perderse de vista el origen autobiográfico de la novela que, no obstante, sería matizado convenientemente por el propio Cela en los sucesivos prólogos que dedicó a las distintas ediciones de Pabellón 
de reposo..$^{5}$ Dos son las experiencias sanatoriales vividas por Cela que sirvieron de punto de partida para la novela del 43: la primera en 1931, cuando Cela contaba 15 años, en el Real Sanatorio de Guadarrama, dirigido por el Dr. Partearroyo; y en 1942, recién finalizada La familia de Pascual Duarte, con 26 años, en el Nuevo Sanatorio Antituberculoso de Hoyo de Manzanares, dirigido por el Dr. Valdés Lambea (Cela 1962b; Moyano). Debido a los juegos entre lo factual y lo ficcional que el propio Cela favorece en Pabellón de reposo, algunos críticos se han apoyado en la base autobiográfica de la novela para ver en el número 52 un alter ego de Cela; tendencia -aunque mirada con reticencianatural y comprensible a ojos de Foster $(37,45)$, McPheeters (52) o García de Nora (56), a la luz de las pistas sembradas en la novela. Otros han intentado, por su parte, rastrear a Cela a través de la multiplicidad de voces que representan los siete personajes de la novela, como es el caso de Ignacio Agustí, quien, en una de las primeras reseñas que recibió la novela, expresaba su consideración de que los personajes de Pabellón no se habían independizado de su autor y que "uno tras otro iban resultando, sin embargo, espectros velados de él” (Agustí 12).

Como hemos avanzado, Camilo José Cela abordó el tema en varias ocasiones, reiterando la misma idea en todas ellas. En Pabellón de reposo recogía "una experiencia personal que marcó en mis días una señal indeleble y venenosa" (1962a, 589); "fue pensada sobre amargas experiencias personales" (1962a, 591); "inmediato producto de una amarga y aleccionadora experiencia personal” (1962a, 205). En el prólogo a la sexta edición de la novela, en 1960, titulada justamente "La experiencia personal de Pabellón de reposo", añadía que su novela era resultado de la fusión de realidad y "situación imaginada" (Cela 1962a, 206). El sanatorio donde transcurren sus últimos días los personajes de Pabellón de reposo constituía una prueba de lo afirmado, puesto que, aun sin corresponderse con ninguno de aquellos dos en los que estuvo Cela, "tiene no poco de ambos" (Cela 1962a, 207).

5. Los textos imprescindibles donde Cela expone sus pensamientos en torno a su novela Pabellón de reposo son: "Nota" a la edición de 1944 en Afrodisio Aguado (Cela 1962a, 587); "Nota a esta edición", la de Destino de 1952 (a la que llama segunda, o tercera si cuenta la edición en prensa, o cuarta si cuenta la edición en 1943 de 25 ejemplares), fechada en Los Cerrillos, sierra de Guadarrama, setiembre de 1952 (Cela 1962a, 588-90); "Nota a lo publicado en Mis páginas preferidas" (A la primera edición: Madrid: Gredos, 1956) (Cela 1962a, 591-92); el Prólogo a Mrs. Caldwell babla con su bijo (1953): "Algunas palabras al que leyere" (Cela 1958, 9-15); "La experiencia personal de Pabellón de reposo" (Nota a la sexta edición), fechado en Palma de Mallorca, 24 de agosto de 1960 (Cela 1962a, 205-08). 
Sin embargo, más allá de la posible autoficcionalidad de la obra, lo que destaca el autor y lo que demuestra la novela es la voluntad de ofrecer al lector una representación; una representación de la enfermedad, de la vida de los enfermos y, sobre todo, de sus narrativas; que busca ser, en palabras de Cela, un "reflejo artístico" de la realidad (1962a, 206). Como todos los autores de narrativas patográficas, el escritor adopta una postura con la que enfrentarse a su narrativa/representación de la enfermedad y del sufrimiento que esta lleva consigo. En 1960 Cela expone la suya cuando afirma, al evocar Pabellón de reposo: "Los tipos están literaturizados -esto es: aguados- porque me pareció excesivo llevar a la página escrita la ruindad, la vileza y la violencia de las que mis atónitos ojos de entonces fueron testigo" (1962a, 207). Este deseo expreso de tamizar la crueldad de la tuberculosis es quizá donde radica la poetización que envuelve las páginas "dulcemente amargas y sin consuelo" (1962a, 589) que componen la narrativa patográfica de Cela. Los remanentes románticos de la antigua imagen de la tuberculosis están presentes en la representación que el autor realiza del mundo de los enfermos. Se hallan en la configuración del número 14 con los rasgos arquetípicos del poeta romántico, a partir sobre todo de la imagen que de él nos ofrece el número 40: "Los ojos encendidos, la sonrisa amarga, la tez pálida, la nariz afilada... Semejaba una estampa romántica, una bella y desusada estampa de daguerrotipo romántico" (63). ${ }^{6}$ El muchacho del 14 se nos presenta como un "Apolo tuberculoso y pudibundo" (165) que recita versos de Nicomedes-Pastor Díaz, y que desearía ser John Keats para contar con su propio Adonais, el conjunto de versos que el también poeta y amigo Percy B. Shelley dedicó a Keats a su muerte por tuberculosis: "Me gustaría haber sido amigo de Shelley para que a mi muerte cantara: «Llorad por él, aunque el ardiente llanto no deshaga la nieve que le cubre»" (156). Las huellas románticas de la tuberculosis se encuentran en el texto celiano igualmente en las referencias literarias a dos mujeres que contribuyeron en el siglo anterior a la correspondencia entre tuberculosis y belleza femenina ("belleza tísica"): Mimí, uno de los personajes de Scènes de la vie de bohème (1845-49 [Scènes de la bohème 1851]), de Henri Murger, y Margarita (Marguerite Gautier), la protagonista de La dame aux camélias (1848), de Alejandro Dumas, hijo; también recordada en otras narrativas sanatoriales, como El balcón hacia la muerte, del argentino Ulises Petit de Murat (13-14).

6. Todas las citas de Pabellón de reposo que se recogen en el cuerpo del texto remiten a la edición en Destino de 1965, convenientemente referenciada en la bibliografía final. 
Romántica es también, en especial, la representación de la buena y dulce muerte con la que los enfermos de Pabellón de reposo acaban sus días. Curiosamente será el número 14 el que admita que "los últimos instantes de los tuberculosos no son, en verdad, tan hermosos como han querido presentárnoslos los poetas románticos" (157). Aun así, en los últimos estertores de la vida de sus personajes, cuando estos han alcanzado la resignación, Cela nos ofrecerá la belleza y la calma de la muerte. El 37 parecerá "una figurita de marfil, con sus alabastrinas manos cruzadas sobre el regazo como en oración, y sus ojos cerrados dulcemente a la vida, como gozosos de haber vuelto a encontrar -jal fin!- la dicha" (131). Los propios enfermos de Pabellón de reposo han interiorizado la dulzura de la muerte reposada cantada en los libros románticos, y esa es la imagen que ofrecen sus discursos. Queda patente de forma clara en la visión del 40:

Por eso amo la luz de las bombillas, la luz que relumbra como el diamante cuando la miramos con los ojos semientornados, suavemente semientornados por el bello cansancio de las tres de la mañana, cuando ya hemos bebido y bailado hasta hartarnos y cuando ya la risa y la conversación van muriendo poco a poco, imperceptiblemente, casi con dulzura, como dicen los libros románticos que morimos los tuberculosos cuando nuestra vida llega ya a esa alta madrugada tan difícil de remontar... (66)

Asimismo, cuando el número 2 siente cercano el final, la muerte es contemplada incluso con complacencia, una muerte "ensoñadora y dulce que solo los poetas, los músicos y a veces las señoritas solteras consiguen de la Providencia" (108).

No se debe pasar por alto que cuando los enfermos de Pabellón de reposo reproducen esta muerte tranquila de la literatura romántica no es solo herencia de la imagen estilizada que se tenía de la tuberculosis en el siglo XIX, sino también de la misma idealización que de ella vislumbraba el autor en la "Nota" a la edición de 1952, al recordar la inacción de la novela:

Como las drogas, el reposo llega a ser un vicio del que no es nada fácil sustraerse una vez iniciados en él. Y como las drogas, el reposo es también un mundo deleitoso y lento en el que la ansiada meta se confunde con el diluirse, como un brevísimo terrón de azúcar, en el mar inmenso de la muerte, un mar que nos ofrece una muerte que no duele y que se nos presenta vaporosa y puntual como una recién casada en pecado. (Cela 1962a, 589) 
Por otro lado, los personajes, en referencias cruzadas (nueva muestra de cómo las distintas narrativas que componen la novela se llaman entre sí), ven al otro como el soñador o el romántico, y sus discursos, configurados a menudo con estructuras trimembres de adjetivos (como la angustiante alusión a las "pobres, tristes, doloridas carnes" del número 52 [127]), podrían desgajarse a veces en versos becquerianos: "Aquellos amores no correspondidos, aquellos hermosos proyectos que el tiempo se encargara de enfriar, y después de echar por tierra, ¡quién pudiera volverlos a añorar!” (141). Esta tendencia poetizante la achacaba el Dr. Leopoldo Cortejoso precisamente a la imposibilidad de sujetar la fantasía ante tanto reposo. De esa intensidad con la que vive su vida interior el enfermo nacía -a juicio del autor de Tuberculosos célebres- el pensador, el idealista, el poeta (Cortejoso 1939): "¿Y cómo no creer que la enfermedad [de la tuberculosis] tiene su parte de culpa en la aventura poética o al menos en la forma de manifestarse?" -se preguntaba, afirmando, en 1958-(Cortejoso 1958b, 16).

La presente poeticidad, sin embargo, no resta crueldad a la enfermedad de los tuberculosos del pabellón, de quienes nos llegan sus continuos sufrimientos, que no son poesía, sino realidad. Si bien pedía perdón Cela en la "Nota" a la edición de 1952 "por disfrazar la ternura de crueldad" (1962a, 590), la sensación que procura al lector Pabellón de reposo gracias al lirismo que recorre la narrativa es, por el contrario, que Cela desea disfrazar la crueldad de ternura. No obstante, el silencio, la tamización de la crueldad y el efecto difuminado elegido, así como la explicitación como autor de su elección tienen la función de acentuar, por oposición, la cruda realidad del enfermo del sanatorio; realidad que nos llega vehiculada principalmente -además de las interrupciones que subrayan la base real de la novela- a través del relato de los propios enfermos.

La esencia de las narrativas patográficas, también la de Cela, radica en dos cuestiones básicas de carácter circunstancial: el para qué y el por qué se escribe, ambas incluidas en el discurso de Pabellón de reposo.

En cuanto a la primera pregunta, con qué finalidad se escribe la narrativa, resulta llamativo cómo en la década de los cuarenta Cela aborda en su novela, tempranamente, aunque sea de manera tangencial, un asunto que ocupa a la disciplina de las Medical Humanities (ver, entre otros, los estudios de Cousins, Kleinman, Frank, Hunsaker Hawkins, Keen, Juracic o Whitehead) desde su surgimiento: la posible práctica reparadora o, por el contrario, el efecto negativo que pueden tener las narrativas patográficas para el yo-autor-enfermo (más claro en las autopatografías), o para otros lectores que comparten (di- 
recta o indirectamente) la enfermedad y que, por lo tanto, leen la patografía desde la identificación.

Como recordará el lector de Pabellón de reposo, en la interrupción que como autor realiza en la obra, Cela ficcionaliza la segunda de las posturas al recoger las críticas que ha recibido acerca de que su novela -para evitar el daño que pueda ocasionar en los enfermos- sea distribuida/leída en los sanatorios, e incluso haya sido prohibida, según declara en 1952, en la "Nota" para la edición de Destino: "Me anima a ello [a reeditar la novela] [...] el hecho de que el libro esté prohibido en los sanatorios antituberculosos" (1962a, 590). En este contexto, cobran sentido las reticencias del editor Lara en 1951 a publicar la novela leída por él y su mujer, y que fue desestimada "porque no me atrevo a publicar una novela que deje abatidos a los lectores" (Sotelo 31).

Dicha postura choca curiosamente con la impresión del que fuera el propio médico de Cela en su vivencia sanatorial en Hoyos de Manzanares, José Valdés Lambea. Este médico especialista en tuberculosis pulmonar, director de varios sanatorios, además del de Hoyos, autor de varios trabajos médicos en torno al tratamiento de la enfermedad y director de la Revista Española de Tuberculosis, publica en 1944 un librito pseudo-literario titulado Vidas de sanatorio (tipos, ocurrencias y conversaciones), que con tintes de novela romántica, ficcionaliza el microcosmos del sanatorio, donde las conversaciones giran en torno a la tuberculosis misma y a aquellos escritores célebres que la padecieron. En esta nueva pieza de literatura sanatorial que Valdés Lambea termina de escribir en enero de 1942 (por tanto, poco antes de entrar Cela en el sanatorio), se defiende el beneficio para el enfermo que puede tener la literatura tuberculosa. El volumen de Lambea, calificado en el primer capítulo como "el libro del Sanatorio Antituberculoso" (Valdés Lambea 6) nace explícitamente con un "propósito de instruir" (7), mediante un libro "hecho por un tuberculoso, pensando en los tuberculosos y para sus compañeros de hermandad" (10). Asimismo, en el sanatorio recreado por Valdés Lambea los doctores comentan con naturalidad y aceptación los varios ejemplares que de La montaña mágica (referencia y modelo claros de Valdés para la composición de su propia obra) "andan rondando [...] por el sanatorio" (51). Para ser justos con este beneplácito de La montaña mágica con el que, por el contrario, no parece contar en el mismo periodo Pabellón, no se puede sortear que Cela acentúa el lado más sombrío de la tragedia del tuberculoso, al dejar poco espacio al optimismo, presentando a unos enfermos que, lejos de entregarse a alegres comidas o excursiones (escenas frecuentes en La montaña mágica), deben hacerlo a la de- 
sesperanza y a la resignación de verse incapaces ya de sacar la cama de ruedas a la galería, y donde absolutamente todos pasarán a formar parte de las estadísticas del sanatorio de bajas por defunción.

Por otra parte, es de igual modo sugestiva la presentación en el número 6 de La Estafeta Literaria (31 mayo 1944), con motivo de la publicación de Pabellón de reposo en volumen, de tres artículos bajo el título general "Consulta de médicos alrededor de Cela". En lo que supone un nuevo reflejo del interés por parte de la Medicina hacia las Humanidades, tres médicos analizan someramente la relación entre ciencia y literatura que podía rastrearse en las entonces dos últimas novelas de Cela. Mientras que el doctor López Ibor manifestaba su atracción hacia el carácter patológico del protagonista del Pascual en "Psicopatía y literatura", los doctores Manuel Izquierdo Hernández y Rafael Romero Moliner se ocupaban en sendos artículos de la representación que ofrece Cela de la tuberculosis. Es especialmente relevante para el tema que nos ocupa la visión del primero, cuando -doctor él mismo- critica a los compañeros de profesión representados en la novela, quienes -centrados en lo biomédico- se revelan ajenos a cómo afecta a la sensibilidad del paciente la enfermedad y, por tanto, indirectamente, se olvidan de su narrativa (algo que, en cierto modo, claman las Medical Humanities):

Los enfermos que describe Camilo José Cela [dice el Dr. Izquierdo] son tratados por tisiólogos, maquinistas de la técnica, que muchas veces solo perciben la lesión anatómica sin preocuparse de la constitución, de la gerencia, de las alteraciones que en la psicología imprime la enfermedad. (VV.AA. 1944, 9)

Cela lleva a su novela del 43 esta doble perspectiva que suscitaba la literatura sanatorial. El motivo del daño que la identificación con el enfermo podría causar en el lector, enfermo a su vez, le sirve para llevar a cabo el procedimiento literario de interrumpir el discurso narrativo, en tanto autor, en dos ocasiones. $\mathrm{Ni}$ los consejos acerca de poner fin a su obra (en marcha en ese momento en las páginas de $E l E s p a \tilde{n} o l)^{7}$ que le llegan de un conocido tisiólogo, y más tarde de un amigo sufridor de la enfermedad hacen cala en Cela. El autor elegirá continuar, defendiéndose con el carácter ficcional de sus personajes. No cabe la identificación, puesto que el tipo de personaje "ombligo del mundo" (Cela

7. Antes de publicarse en volumen en 1944, Pabellón de reposo vio la luz por entregas en El Español, desde el 13 de marzo de 1943 hasta el 21 de agosto de 1943. Ver Foster 34. 
$1965,96)$ por él pintado es precisamente aquello de lo que debe huir el enfermo tuberculoso, y ahí está el posible beneficio de su novela. Aún en 1952, cuando ya la curación de la tuberculosis es un hecho, a Cela le siguen asaltando las dudas acerca de silenciar caritativamente una nueva edición de sus narrativas patográficas. Como en 1943, se decide a seguir también esta vez, sabiendo que "los tuberculosos gozan con su lectura" (Cela 1962a, 590).

En relación con la segunda cuestión que mencionábamos más arriba acerca de las razones que hay detrás de la escritura de una narrativa patográfica, es oportuno recordar que, quizá como herencia de la tradición romántica de la tuberculosis que pervive aún en el siglo XX, algunos renombrados médicos especialistas defenderán, todavía en la década de los cuarenta, una conexión entre la tisis y la creación artística. El Dr. Leopoldo Cortejoso, por ejemplo, que como Valdés Lambea había escrito no solo varias obras médicas acerca del tratamiento de la enfermedad, sino que también había realizado una incursión en la literatura publicando el mismo año que Pabellón de reposo el volumen El dolor en la vida y el arte: ensayos médico-biográficos sobre tuberculosos célebres (1943), defendía en 1939, al ocuparse del papel de la enfermera en la lucha antituberculosa, que la intoxicación tuberculosa estimulaba las facultades creadoras del enfermo, de manera que surgía el afán de materializar lo que estaba en el pensamiento, y por lo tanto, se fomentaba el espíritu. Por otra parte, Cortejoso sostiene que la tuberculosis influyó poderosamente sobre la actividad creadora de famosos artistas dominados por la enfermedad, quienes produjeron sus mejores obras precisamente cuando la tisis actuaba con más violencia y consumía con más fuerza el organismo (Cortejoso 1939). Estas opiniones suponían, además, la contribución del Dr. Cortejoso a la recuperación continua que se lleva a cabo, en la década de los treinta y cuarenta, de aquellos artistas que sufrieron tuberculosis; origen, en parte, de la revitalización que se produce en el siglo xx del perfil estilizado de la enfermedad (la mayor parte de los tuberculosos célebres que se rescatan son del siglo XIX). ${ }^{8}$

8. En 1944 aparecía no solo el volumen ya citado de Valdés Lambea (Vidas de sanatorio. Tipos, ocurrencias y conversaciones), que dedica algunas de sus páginas a la memoria de artistas tísicos, sino que también veía la luz la traducción al español de la obra de Lewin J. Moorman, Tuberculosis and genius (1940), con el título La tuberculosis en los grandes genios. Por su parte, Cortejoso continúa en 1958 la línea de su obra del 43 sacando a la luz Tuberculosos célebres: grandes personalidades forjadas por la tuberculosis. Resulta especialmente sugerente la disertación de Cortejoso acerca de la estrecha relación entre enfermedad y poesía, que desemboca en dos textos curiosos: "Presencia de la tisis en la poesía lírica" (1950), y "La aventura poética en el hombre enfermo" (1958b). 
En suma, el Dr. Cortejoso relaciona la hipersensibilidad que caracteriza al enfermo, y el énfasis que alcanza su vida interior, con la necesidad de la escritura y de la comunicación, que él, referente en España en la lucha antituberculosa, no reprueba. No así el médico que elige Camilo José Cela para su representación en Pabellón de reposo, que acentúa la distancia entre médico y paciente, y por tanto la oposición elemental entre el mundo de los enfermos y el mundo de los sanos. En el informe presentado a los accionistas en el Intermedio de la novela, el médico residente se detiene en la relación entre enfermedad y literatura cuando incide en la pasión con que los pacientes se entregan a la escritura de sus diarios y memorias. En lo que supone una postura tradicionalista (poco humanista, podríamos decir) sobre el valor de las narrativas para el enfermo, el doctor se planteará la ruptura de dicho vínculo:

Hemos observado que crece el número de desequilibrios nerviosos entre nuestros clientes. Las causas las ignoramos. Hemos observado también que casi todos aquellos clientes en quienes hemos visto esos trastornos se dedican a escribir con toda pasión sus diarios o sus memorias. Pienso que quizás haya llegado el caso de aconsejarles que abandonen la literatura. (114)

Sin embargo, la escritura aflora como una necesidad de comunicación en el enfermo, sobre todo el crónico. Mientras que la salud nos impone el tiempo de la actividad y la acción física corresponde al cuerpo sano, la enfermedad inmoviliza, el cuerpo enfermo se ve apartado de la acción, y el movimiento se desplaza del cuerpo a la mente; pensamos, hablamos, y en ese tiempo dilatado de la inacción, las palabras adquieren todo su poder de alusión, a veces terrorífico. El enfermo, aislado, exiliado en sí mismo, buscará un interlocutor en su propio yo. La narrativa patográfica, nacida del aislamiento y la incomunicación con el otro, potencia la introspección, la conexión con el yo; y Pabellón de repo$s o$, en relación con otras narrativas sanatoriales, supone una mayor intensificación del mundo interior de los protagonistas. A diferencia de lo que ocurre en las obras de Thomas Mann (Der Zauberberg, 1924), Dino Buzzati (Sette piani, 1937) o Gesualdo Bufalino (Diceria dell'untore, 1981), apenas se describe el espacio físico del sanatorio, y los tuberculosos de Cela apenas se mueven, apenas se relacionan, y las conversaciones existentes le llegan al lector fundamentalmente en estilo restituido, pues importan más las impresiones que de la conversación tienen sus interlocutores. Contrariamente a otras representaciones de sanatorios, en la novela de Cela "no se atiende [en palabras del autor] sino a sus estertores, a las últimas luces de cada candil” (Cela 1958, 13). De ahí que 
en sus últimos resuellos, a punto de apagarse sus vidas, el autoanálisis y el pensamiento recurrente en torno al "yo" sea, como lo será para Hervé Guibert en los años noventa, un refugio, en forma de la escritura de cartas, diarios, o en forma de diálogos y monólogos interiores. ${ }^{9}$

Esas serán las distintas formas en las que se materialicen las siete narrativas patográficas de las que se compone Pabellón de reposo, distribuidas, según una estructura perfectamente simétrica detallada ya por Ilie o Foster, en dos partes separadas por un Intermedio que ayuda a proporcionar la sensación del tiempo transcurrido, cronológico y psicológico, entre unas y otras intervenciones. Además, este Intermedio, con la presentación de breves escenas de la vida activa del sanatorio, paralela a los internos, tiene la función de subrayar la oposición, propia de la literatura sanatorial, que se establece entre el mundo de los sanos y el mundo de los enfermos. Tanto el personal médico como el de servicio mantienen una brecha emocional con el paciente, al que han cosificado, subrayando así el exilio del tuberculoso. Lo cosifica la enfermera que, "sana y robusta", se ríe del paciente al que la muerte ha encontrado desnudo y bañado en sangre; y lo cosifican los médicos cuando los conciben como "clientes", números de estadísticas, bajas, cifras.

La escritura será el medio por el que se materialice la necesidad de comunicación que se apodera del enfermo, enclaustrado en el espacio del sanatorio, pero también en su propio cuerpo enfermo. Las cartas, los diarios, los momentos de monologar con uno mismo, parecen la única salida que le queda al interno tras el proceso de desposesión que supone la admisión en el sanatorio. Al proceso de deconstrucción que supone la pérdida de la identidad de la entrada al sanatorio (pérdida del nombre; marcación de la ropa; reducción de espacios íntimos; cirugía mutilante, deformadora y por tanto desposeedora; la limitación del movimiento; convertirse casi en un títere en manos del personal), ${ }^{10}$ le sigue inevitablemente un proceso de construcción del "yo enfermo". Para ello, es necesaria una autobjetivación, una especie de cosificación del yo, por medio de la cual el "yo" no solo será sujeto, sino al mismo

9. Para Hervé Guibert, autor de la autoficción À l'ami que ne m'a pas sauvé la vie (1990), la escritura patográfica a la que le lleva el sida es vista como un refugio: "[...] moi qui viens de découvrir que je n'aime pas les hommes, non, décidément, je ne les aimes pas, je les haïrais plutôt, et ceci expliquerait tout, cette haine tenace depuis toujours, j'entreprends un nouveau libre pour avoir un compagnon, un interlocuteur, quelqu'un avec qui manger et dormir, auprès duquel rêver et cauchemarder, le seul ami présentement tenable" (Guibert 12).

10. El episodio de la enfermera riéndose ante el espectáculo de la muerte de uno de los pacientes será el punto culminante de esos enfermos-marionetas. 
tiempo objeto de sí mismo. En el momento en que el yo se distancia, aun involuntariamente, del objeto externo; es decir, de la vida más allá de los límites del sanatorio, donde se adivinan las luces de la ciudad, y de la vida activa dentro de esos límites (médicos, enfermeras, servicio); en el momento en que se rompen los últimos amarres, debe cambiarse el objeto de atención. Ahora el objeto es otro, y como consecuencia de esa sustitución, la enfermedad se convertirá en centro de las preocupaciones y obsesiones. En ese proceso de reconstrucción, en cierto modo identitaria, el lector asistirá a la evolución del personaje (a nivel anímico) de acuerdo con los cambios que la enfermedad produce en su cuerpo a nivel físico, hasta tomar conciencia de su propio final. El hecho de que esta restauración del "yo" vaya acompañada paralelamente del desgaste corporal que no otro es el significado de la palabra consunción (un desgaste constante en la novela), propicia que Cela nos presente una evolución psicológica que nos permite asistir a la desintegración de las fuerzas vitales, a los últimos estertores, a la necesidad de reprimir el deseo, a las últimas esperanzas disipadas, para aceptar lo inevitable, para alcanzar la resignación.

El foco temático de esta comunicación con uno mismo será la enfermedad. El estado del cuerpo, su desintegración, determina los derroteros de la narrativa. La muerte envuelve todas las cartas, los diarios, los monólogos, las confesiones. Su presencia es constante a través del simbolismo de la eterna carretilla en Pabellón de reposo, de la camilla chirriante en El mar, o del trineo en La montaña mágica. Es una muerte lenta y acechante, y como tal se metamorfosea en un acreedor que pasa factura cobrándose los pagarés en forma de horas de reloj, como en Clarín ("y se figuraba que la hora, sonando con aquella solemnidad, era como la firma de los pagarés que iba presentando a la vida su acreedor, la muerte" [571]), o en forma de libras de vida a la manera de un eterno Prometeo o de un minotauro que exige su nueva presa, como en Bufalino ("Un re forestiero m'era venuto ad abitare sotto le costole, un innominabile minotauro, a cui dovevo giorno per giorno in tributo una libbra della mia vita" [15-16]). Cuando en Pabellón de reposo la muerte está lejos de la dulcificación romántica y es vista en toda su monstruosidad, el enfermo de tuberculosis intenta definir su última agonía también mediante metáforas que tienen en común el lento y amargo proceso de deterioro, y que ayudan a construir culturalmente, según la idea clásica de Susan Sontag, la imagen de la enfermedad. Para el 37, su tuberculosis es "un mal terrible y pegadizo [que] le come las entrañas" (39); el 11 siente cómo la muerte le 
"consume el pecho" (79) y el final está representado por "el reloj que marcha, sin piedad alguna, sin consideración de ninguna clase, a dejarnos abandonados en cualquiera de las horas, como esos navegantes que se caen al mar desde la borda de los transatlánticos, en mitad del océano" (73) sin que nadie se inmute. Para el 52, la espera, el final seguro pero incierto está simbolizado por la muerte escondiéndose "para atacarnos, para hacer aún más segura presa" (127).

Circunscrito a unos límites muy reducidos, tanto espaciales como temporales, el enfermo se ve obligado a romper dichos límites volviendo la mirada hacia adentro. Cuando la desconsoladora prognosis de la enfermedad antes de 1944 hace que el futuro sea incierto y el presente inestable y devorador, solo el pasado parece un ancla posible. Las únicas salidas que le quedan al enfermo para salir de su escafandra, ${ }^{11}$ además de la mirada (ver Cela 1978) desde las galerías abiertas de los pabellones o las ventanas de la habitación, son la imaginación y la memoria, ${ }^{12}$ como lo eran también para Mrs. Caldwell en el espacio de su casa o del hospital de lunáticos. Mirada y memoria, pues, de nuevo como los dos pilares elementales de la obra celiana. ${ }^{13}$

Por la desnudez abrumadora de los "interiores ahumados" que nos presenta Cela en Pabellón de reposo, donde el mundo externo pasa a un segundo plano, y el espacio alienante del sanatorio con su tiempo detenido promueve la introspección, el autoanálisis y la confesión de los personajes, algunos críticos destacaron como rasgos definitorios de la novela su lirismo y su subjetividad. Según José María Castellet, Pabellón de reposo podía clasificarse, como Mrs. Caldwell habla con su bijo, dentro de las "novelas de intención lírica o de la subjetividad" (44); Gonzalo Sobejano la incluía entre las novelas de confesión (66-67); y García de Nora la calificaba de "novela casi «lírica» y aparentemente inmovilista, estática, una especie de elegía, de adiós a la vida, entonado por varios sujetos, pero todos ellos sometidos a la voz dominante del autor" (55). En cierta manera lo había avanzado el autor cuando en la "Nota" a la edi-

11. Tomo prestada la metáfora del título que Jean-Dominique Bauby le dio a su narrativa patográfica publicada en 1997, Le scaphandre et le papillon, surgida tras sufrir el locked-in syndrome (síndrome del cautiverio), que le paralizó completamente el cuerpo (a excepción del ojo izquierdo), pero le mantuvo las facultades mentales intactas.

12. Es lo único que le queda intacto a Jean-Dominique Bauby. El recuerdo será el motivo obsesivo para el protagonista tuberculoso de "Ester Primavera" (Arlt), pensando en la antigua amante a lo largo de los setecientos días que lleva ya en el pabellón.

13. La mirada y la memoria han vertebrado los sucesivos estudios que sobre la obra de Cela ha realizado el especialista Adolfo Sotelo. 
ción de 1952 afirmaba que su obra del 43 tenía tanto "de novela como de poema en prosa" (Cela 1962a, 589), o cuando había decidido tamizar la realidad representada, alejándose en cierto modo de lo feo de la enfermedad, poetizando la tuberculosis.

Pabellón de reposo responde en todo caso al tipo de novela que por esos años decía buscar su autor, cuando defendía en un artículo titulado "Sobre el concepto de novela", publicado en $\mathrm{Haz}$, en febrero de 1943, apenas un mes antes de dar la primera entrega de su nueva novela en El Español:

La novela precisa de una verdad entrañable, de una verdad de cuerpo entero, de una verdad muy digerida por su autor. El novelista debiera tener cuatro estómagos, como los bueyes: panza, bonete, libro y cuajar. Con un sistema así estaría siempre rumiando esa verdad y la novela saldría mejor, más acabada. Con cuatro estómagos no hay quien se atreva a hacer equilibrios. (Cela 2016, 87)

A pesar de la poca atención que ha recibido por parte de la crítica en comparación con el resto de su obra narrativa, Pabellón de reposo tiene el valor de ofrecernos esa verdad entrañable; es decir, surgida de las entrañas; resultado de haber buceado en los interiores del alma enferma, de la suya, pero también de la del otro, y habernos dado la verdad de su narrativa patográfica, aunque la memoria y las raíces románticas de la enfermedad se hayan encargado de cribar esa verdad.

\section{OBRAS CITADAS}

Agustí, Ignacio. "Sobre el Nuevo Lazarillo y sobre Camilo José Cela". La Estafeta Literaria 16 (15 noviembre 1944): 12.

Alas, Leopoldo (Clarín). "El dúo de la tos". Obras Completas, III: Narrativa breve. Ed. Carolyn Richmond. Oviedo: Ediciones Nobel, 2003. 569-74.

Arlt, Roberto. "Ester Primavera". El jorobadito. Buenos Aires: Compañía General Fabril Editora, 1968. 71-89.

Bauby, Jean-Dominique. Le Scaphandre et le papillon. Paris: R. Laffont, 1997. Bonet, Blai. El mar. Barcelona: Aymà, 1958.

Bryder, Linda. Below the Magic Mountain: A Social History of Tuberculosis in Twentieth-Century Britain, 1876-1938. New York: Clarendon Press Oxford/Oxford UP, 1988.

Bufalino, Gesualdo. Diceria dell'untore. Palermo: Sellerio, 1981. 
Burgos de Pablo, Abilio. "Apuntes para el estudio de la tuberculosis en Palencia, en España y en el mundo". Publicaciones de la Institución Tello Téllez de Meneses 49 (1983) 145-239.

Buzzati, Dino. "Sette piani”. 1937. Sessanta racconti. Milán: Mondadori, 1963. 31-51.

Castellet, José M. a . "Iniciación a la obra narrativa de Camilo José Cela”. vV.AA. Camilo fosé Cela: vida y obra-bibliografía-antología. New York: Hispanic Institute in the United States, 1962. 7-50.

Castro, Rosalía de. "A Manuel Murguía”. Cartas. Alicante: Biblioteca Virtual Miguel de Cervantes, 2002. 30 de marzo de 2016. <http://www.cervantesvirtual.com/obra-visor/cartas--1/html/feed6a52-82b1-11df-acc7002185ce6064_2.html\#I_1_>.

Cela, Camilo José. "Sobre el concepto de novela". La forja de un escritor (19431952). Ed. Adolfo Sotelo Vázquez. Cuadernos de obra fundamental. Madrid: Fundación Banco Santander, 2016. 85-87.

Cela, Camilo José. Pabellón de reposo. 1944. Barcelona: Destino, 1965.

Cela, Camilo José. "Algunas palabras al que leyere”. Mrs. Caldwell habla con su bijo. 1953. Barcelona: Destino, 1958. 9-15.

Cela, Camilo José. Obra Completa, I: Las tres primeras novelas (1942-43-44). Barcelona: Destino, 1962a.

Cela, Camilo José. "Relativo currículum vitae". vv.AA. Camilo fosé Cela: vida y obra-bibliografia-antología. New York: Hispanic Institute in the United States, 1962b. 79-109.

Cela, Camilo José. "Esa ventana abierta sobre cualquier paisaje". Arriba 5 septiembre 1950. Glosa del mundo en torno. Obra Completa. Vol. 10. Barcelona: Destino, 1978.

Cortejoso, Leopoldo. La enfermera en la lucha antituberculosa. Valladolid: Librería Santarén, 1939.

Cortejoso, Leopoldo. El dolor en la vida y en el arte: ensayos médicobiográficos sobre tuberculosos célebres. Barcelona: Iberia, Joaquín Gil Editor, 1943.

Cortejoso, Leopoldo. Presencia de la tisis en la poesía lírica. Conferencia. Madrid: Asociación Española de Escritores Médicos, 1950.

Cortejoso, Leopoldo. Tuberculosos célebres: grandes personalidades forjadas por la tuberculosis. Barcelona: Mateu, 1958a.

Cortejoso, Leopoldo. La aventura poética en el hombre enfermo: de Bécquer a fosé Luis Hidalgo. Discurso leído ante la Real Academia de Bellas Artes de la Purísima Concepción de Valladolid. Valladolid: Minerva, 1958b. 
Cousins, Norman. Anatomy of an Illness as Perceived by the Patient: Reflections on Healing and Regeneration. New York: Norton, 1979.

Dubos, René Jules y Jean Dubos. The White Plague: Tuberculosis, Man and Society. Boston: Little Brown, 1952.

Foster, David W. "Pabellón de reposo: An Experiment in the Novel of Psychological Introspection". Forms of the Novel in the Work of Camilo Fosé Cela. Columbia-Misouri: Missouri UP, 1967. 34-48.

Frank, Arthur W. The Wounded Storyteller: Body, Illness, and Ethics. Chicago/ London: Chicago UP, 1995.

García de Nora, Eugenio. "Sobre Pabellón de reposo". Insula 518-519 (febreromarzo 1990): 55-56.

Goffman, Erving. Asylums: Essays on the Social Situation of Mental Patients and Other Inmates. Garden City, NY: Anchor Books, 1961.

Goffman, Erving. Stigma: Notes on the Management of Spoiled Identity. New Jersey: Prentice-Hall, Inc., 1963.

Guibert, Hervé. À l'ami qui ne m’a pas sauvé la vie. Paris: Gallimard, 1990.

Holmes, Oliver Wendell. "The Medical Profession in Massachussets". Medical Essays 1842-1882. Boston/New York: Houghton, Mifflin And Company, 1911. 312-69.

Hunsaker Hawkins, Anne. Reconstructing Illness: Studies in Pathography. West Lafayette: Purdue UP, 1999.

Ilie, Paul. "Pabellón de reposo". La novelística de Camilo Fosé Cela. Cap. 2. Madrid: Gredos, 1963. 77-111.

Juracic, Ann. Illness as Narrative. Pittsburg: Pittsburg UP, 2012.

Keen, Suzanne. Empathy and the Novel. Oxford: Oxford UP, 2007.

Kirsner, Robert. "Pabellón de reposo". The Novels and Travels of Camilo Fosé Cela. Chapel Hill: North Carolina UP, 1963. 35-46.

Kleinman, Arthur. Illness Narratives: Suffering, Healing and the Human Condition. New York: Basic Books, 1988.

Kronik, John W. "Pabellón de reposo: la inquietud narrativa de Camilo José Cela". Actas del VIII Congreso de la Asociación Internacional de Hispanistas (Providence 22-27 de agosto de 1983). Vol. 2. Eds. David Kossoff, Ruth H. Kossoff, Geiffrey Ribbans y José Amor y Vázquez. Madrid: Istmo, 1986. $105-11$.

Lawlor, Clark. Consumption and Literature: The Making of the Romantic Disease. Nueva York: Palgrave MacMillan, 2006.

Madkour, M. Monir, ed. Tuberculosis. Heidelberg: Springer-Verlag Berlin, 2004. 
Mann, Thomas. La montaña mágica. 1924. Trad. de Mario Verdaguer. Pról. de Javier Alfaya. 2 vols. Madrid: El Mundo, 1999.

Martín Moyano, Rafael. "Camilo José Cela y Hoyo de Manzanares". La Gatera de la Villa 17 (abril 2014): 66-72.

McPheeters, D. W. "The Story of a Writer". Camilo fosé Cela. New York: Twayne Publishers, Inc., 1969. 15-30.

McPheeters, D. W. "A New Technique”. Camilo fosé Cela. New York: Twayne Publishers, Inc., 1969. 52-61.

Molero Mesa, Jorge. "La tuberculosis como enfermedad social en los estudios epidemiológicos españoles anteriores a la guerra civil". Dynamis: Acta Hispanica ad Medicinae Scientiarumque Historiam Illustrandam 9 (1989): 185-223.

Molero Mesa, Jorge. "Enfermedad y previsión social en España durante el primer franquismo (1936-1951): el frustrado seguro obligatorio contra la tuberculosis". Dynamis: Acta Hispanica ad Medicinae Scientiarumque Historiam Illustrandam 14 (1994): 199-225.

Moorman, Lewin J. La tuberculosis en los grandes genios. Buenos Aires: Guillermo Kraft, 1944.

Navarro García, Ramón. Análisis de la sanidad en España a lo largo del siglo XX. Madrid: Ministerio de Sanidad y Consumo/Instituto de Sanidad Carlos III, 2002.

Pérez, Óscar. "Una aproximación biopolítica a la narrativa de la posguerra: la tuberculosis en Pabellón de reposo de Camilo José Cela y El mar de Blai Bonet”. Hispania 98.2 (junio 2015): 231-42.

Petit de Murat, Ulises. El balcón hacia la muerte. Buenos Aires: Editorial Lautaro, 1943.

Pieltáin Álvarez-Arenas, Alberto. "Los hospitales de Franco: la versión autóctona de una arquitectura moderna". Tesis doctoral. Madrid: Universidad Politécnica de Madrid. Escuela Técnica Superior de Arquitectura, 2003.

Ruiloba Quecedo, Cecilia. Arquitectura sanatoria: sanatorios antituberculosos. Madrid: Escuela Nacional de Sanidad, 2014.

Sobejano, Gonzalo. "Cela y la renovación de la novela". Ínsula 518-519 (febrero-marzo 1990): 66-67.

Sontag, Susan. Illness as Metaphor and AIDS and Its Metaphors. 1978/1988. New York: Picador USA, Farrar, Straus and Giroux, 1990.

Sotelo Vázquez, Adolfo. "Camilo José Cela y los editores barceloneses (19421964)”. La Vanguardia 7 mayo 2016: 28-31. 
Valdés Lambea, José. Vidas de sanatorio: tipos, ocurrencias y conversaciones. Ilustraciones de Luisa Butler. Burgos: Editorial Aldecoa, 1944.

VV.AA. "Consulta de médicos alrededor de Cela". La Estafeta Literaria 6 (31 mayo 1944): 9.

Whitehead, Anne. "The Medical Humanities: A Literary Perspective Overview". Medicine, Health and the Arts: Approaches to the Medical Humanities. Eds. Sam Goodman, Alan Bleakley y Victoria Bates. Abingdon, Oxon: Routledge, 2014. 107-27.

Zamora Vicente, Alonso. "Novela de la inacción". Camilo fosé Cela: acercamiento a un escritor. Madrid: Gredos, 1962. 81-85. 